\title{
What is users' next best alternative to the use of dynamical seasonal predictions?
}

\author{
M. De Felice ${ }^{1} \&$ C. Buontempo ${ }^{2}$
}

\footnotetext{
${ }^{1}$ Agenzia nazionale per le nuove tecnologie l'energia e lo sviluppo economico sostenibile (ENEA), Italy

2 European Centre for Medium-Range Weather Forecasts (ECMWF), UK
}

The recent development of climate predictions has shown their potential in providing users with information that could be used to inform decision on a seasonal time-horizon. This in turns offers a way of developing resilience to climate change through adaptation to seasonal variability.

An effective way to assess to value of this information is to compare the quality of the forecast (assessed by its verification attributes) to the quality of the forecast from a system based on simpler assumption (and thus cheaper to run). For this purpose, climatology and persistence are commonly used. We are presenting here a general methodology based on a Markov Chain process trained at a seasonal time-scale which can be used as a simple benchmark in assessing the skill and thus the value of the predictions.

We demonstrate that in spite of its absolute simplicity our methodology easily outperforms not only climatology but also most of the seasonal predictions system at least in some location such as continental Europe. We suggest that Markov Chain could represent a useful next best alternative for a number of users and thus should be used as a more realistic benchmark.

On a seasonal time-scale empirical models have been used to provide alternative predictions to those generated by dynamical models [1, 2, 3, 4].

More specifically, Markov Chains (MC) [5] have been used in meteorology for a long time [6] as they provide a simple albeit still powerful tool to describe memoryless processes. We developed a simple empirical seasonal prediction based on a Markov Chain model which describes the seasonal variations in 2-metres air temperature (see Methods for details). We adopted a MC approach because its intrinsic simplicity makes it a natural benchmark for dynamical model-based performances whilst offering at the same time an elegant way of addressing the intrinsic state-dependency of climate predictability.

After removing the trend, we have classified temperature values obtained from ERA-20C atmospheric reanalysis at each grid point in three states based on the terciles calculated on a seasonal base. This, by design, makes each state equiprobable. We then calculated the probability of transition from each state to each state of the following season. For simplicity, we limited the analysis to the standard seasons DJF, MAM, JJA, SON but the work can be easily generalised to other seasons. Analysing the transition probability matrix at each location allowed us to identify three different kinds of locations: those characterised by persistence (high 
probability along the main diagonal of the matrix), those characterised by unpredictability (probability values close to 1/3 for most elements) and those characterised by switching (if not semi-periodic) behaviour (high value of probability for elements outside the main diagonal). Following Pasmanter and Timmermann [7] we identify the unpredictable regions as the regions for which the information about the initial state is most rapidly lost. We notice that these regions are also the regions where an effective predictive system has the maximum chance of providing added value, as the trivial option represented here by the Markov chain, doesn't add information over the use of climatological values (i.e. assuming for each state the climatological probability of $1 / 3$ ). Some of the extratropical regions such as some European sub-region in autumn or the Pacific sup-polar gyre areas in spring (Fig. 1) appear to fall in this category.

The persistence-dominated regions instead appear to be characterised by a strong coupling with the slowly varying components of the climate system (e.g. ocean conditions) which provide such a strong constraint onto the atmosphere to significantly influence its seasonal trends and variability. In our analysis, most of the tropics tends to fall in the first second category. This means that if for example the surface temperature of the Nino 3.4 region falls in its top climatological tercile category during SON there are more than $\sim 80 \%$ chances of observing an above average temperature during DJF. For this reason, these are regions where dynamical models are face significant challenges in providing additional value to end users. This is not because dynamical models are somehow deficient or less able to reproduce the key drivers here but because the alternative to the model output is so difficult to beat in these regions. Accounting for the state dependency of the overall predictability and the memory this induces can provide a cost-effective solution to the use a complex dynamical model outputs.

Finally, there are a very few regions (we speculate they could potentially be more common if looking at high-resolution) where switch-behavior is marginally more likely than persistence. For practical applications, these are not too dissimilar from the previous two cases and depending on the specificity of the site what has been said before still applies here. At the same time, some of these locations may offer interesting stimuli for further research as they may highlight areas of instabilities or periodic behavior (see as example the Pacific West Coast of North American as shown in Fig. 2).

In general terms the extreme (i.e. upper and lower tercile categories) appear to be more stable than the central tercile category. The results also show that a two steps transition is unlikely in most location and at least in some of the locations we analysed it has never occurred during the observational record. Similarly, the analysis highlights some of the asymmetries that may exist in the transitions from one state to another.

We have analysed how MC model falls in one of the three categories introduced above and they cluster homogeneous geographical areas. Whilst the spatial distribution of these clusters is neither uniform nor obvious (an example on the North Pacific is shown in Fig. 2), it could provide some clues on the regions where dynamical model outputs are more likely to add value to a simple but not trivial next best alternative.

Surprisingly these regions don't coincide with the regions where models are perceived to perform best (see Fig. 3) as a lot of the model skill seem to depend on simple memory processes that can effectively be modelled statistically.

There are some areas that are worth considering in more details for example the west coast of south America in DJF. In general, the temperature in the Central and South Pacific show a 
strong correlation with ENSO and thus one would assume this could be a region where seasonal prediction models could provide useful insights about the forthcoming climatic conditions. Our analysis shows that not only the trivial model does a decent job in the region but it also improves on the dynamical model output along the cost of Chile where the skill of dynamical models with respect to climatology tend to vanish. This in turns makes that the incremental value of climate predictions from dynamical model negative in the area suggesting that a statistical tool could provide a more than adequate next best alternative to the use of seasonal predictions. South America also provides a nice example of the opposite. Moving east from the coast towards the Andes we can see a region where seasonal model output provides significantly more "value" than the statistical tools. We speculate that this could be linked to the fact that the climate of the region is less strongly constrained by SST and it instead depends also on other drivers and such as snow cover and atmospheric dynamic which are better accounted for by the dynamical models. Up to a certain extent the same applies to eastern Australia another region known for its strong teleconnection to ENSO. Similarly, interesting is the fact that despite the skill, seasonal predictions have recently acquired over the North Atlantic region [12] the MC model in DJF tends to outperform the dynamical model out at least on northern Europe and show similar results elsewhere. More generally Europe appears to be one of the regions where the MC model performs bests in most of the seasons. RPSS of GloSea5, arguably a well-established model in the region, calculated using the MC model as benchmark, shows (see Figure 3) that at present that the predictive value of the numerical-model outputs for users in the region is negative in most cases. At the same time, there are also large areas where the dynamical model outperforms the trivial statistical counterpart (e.g. south-eastern Europe).

Clustering the grid points according to the MC class they belong to (see Figure 4) it is possible to draw some general conclusions about the processes controlling the MC skill. As expected persistence tends to dominate the overall response, particularly true for the tropics during the transition seasons [14]. At the same time the analysis also reveals that there are a number of locations especially in the extra tropics during summer (e.g. Florida and surrounding oceans) and winter (e.g. Scandinavia) where switching behaviour dominates the Markov Chain response. Looking more in-details we have identified some small clusters of strong switching behaviour especially along some coastal areas of North America and Europe. The results are too preliminary and patchy to draw strong conclusions but suggest the possibility that switching behaviour can be more prevalent at higher resolution.

\section{Conclusions}

Climatological frequencies or simple persistence have often been used as a benchmark for seasonal climate predictions derived from dynamical models. Whilst such an approach could represent an efficient way of comparing models with one another it is important to stress that these do not necessarily represent a good proxy for the value the users may see in these predictions. Using a simple MC approach, we have shown that the regions where dynamical model work best tend to coincide with the regions where a simpler alternative also reaches its peak performance. This suggest that the "added value to the users" which depends on the performance of a next best alternative may be maximised in "unexpected" locations characterised by an intrinsic lack of predictability rather than in those locations where well 
established memory processes are more likely to influence the behaviour of the seasonal climate.

The example presented here should also act, in our opinion, as a warning of the possible risks associated with an excessive flattening of the GCM value toward the needs of the users. Assuming that the only metric of interest is the value the predictions could have for decisionmaker could have the counter-productive effect of diminishing the funding towards fundamental research which will still be required in order to develop the next generation of models. We argue that the existence of simple alternative models with similar skill could represent a stimulus for further research whilst at the same time providing a natural benchmark for evaluating more complex kind of predictions. For simplicity of calculations we presented here only the results related to seasonal predictions of temperature. The approach can be easily extended to other time-scale or parameters. 

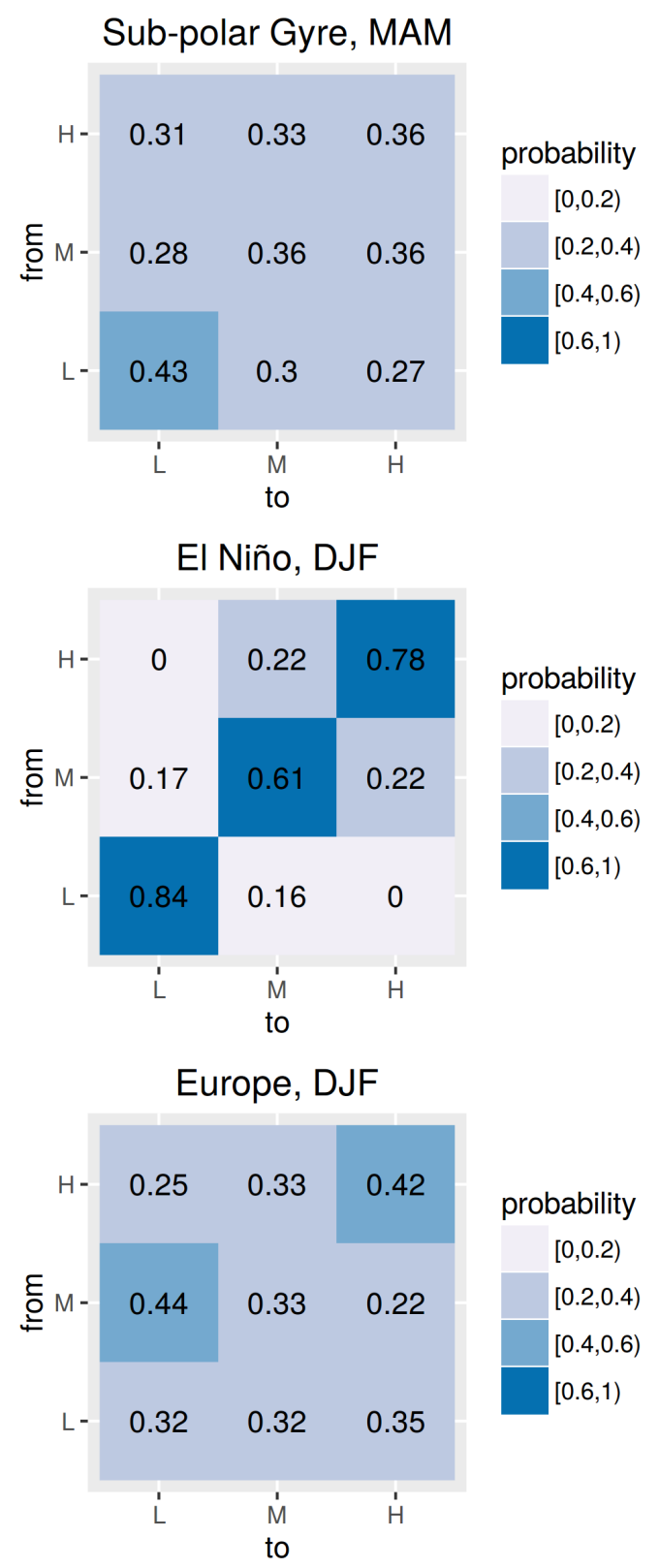

Figure 1. (a) Sub-polar gyre (latitude $40 \mathrm{~N}-65 \mathrm{~N}$, longitude $180 \mathrm{~W}-120 \mathrm{~W}$ ) MC predictions for MAM. The example provides a situation where the evolution of the system represents a nearly perfect random walk. Although for state 1 there is an advantage in assuming persistence assuming no a -priori knowledge for seasonal prediction (and thus a 0.33, 0.33, 0.33 distribution) still represents a reasonable benchmark for seasonal ensemble predictions. (b) Niño 3.4 (latitude 15S-15N, longitude 140W-80W) predictions for DJF. This is a clear example where assuming climatological background as benchmark would be wrong. In most tropical areas and in the Niño region in particular, persistence provides a far better benchmark than any sort of climatology. Particularly interesting is the presence of a zero in the transition matrix. (c) predictions for continental Europe (latitude $35 \mathrm{~N}-60 \mathrm{~N}$, longitude $15 \mathrm{~W}-30 \mathrm{E})$ in DJF. This is the only case we found at this coarse scale for which in at least one state the probability of transition is high than the probability of persistence. 


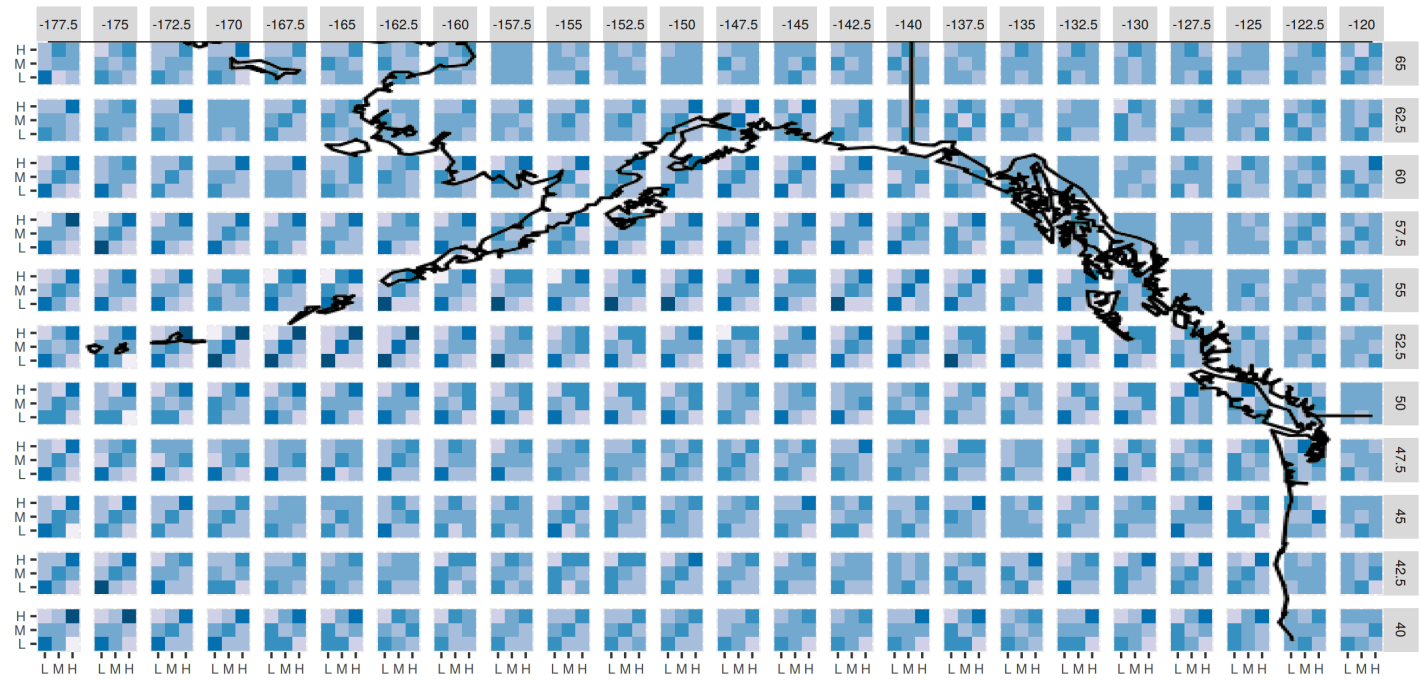

$$
\text { probability } \quad[0,0.1) \longrightarrow[0.1,0.2) \square[0.2,0.3) \square[0.3,0.4) \square[0.4,0.5) \square[0.5,0.6) \square[0.6,1)
$$

Figure 2: a mosaic plot for the probability transition matrix in the north Pacific during Autumn (SON). Different areas can be detected. The central and the western edge of the zone show a remarkable persistence whilst the eastern edge show near perfect random walk conditions. Map created with R 3.3.0 [15]

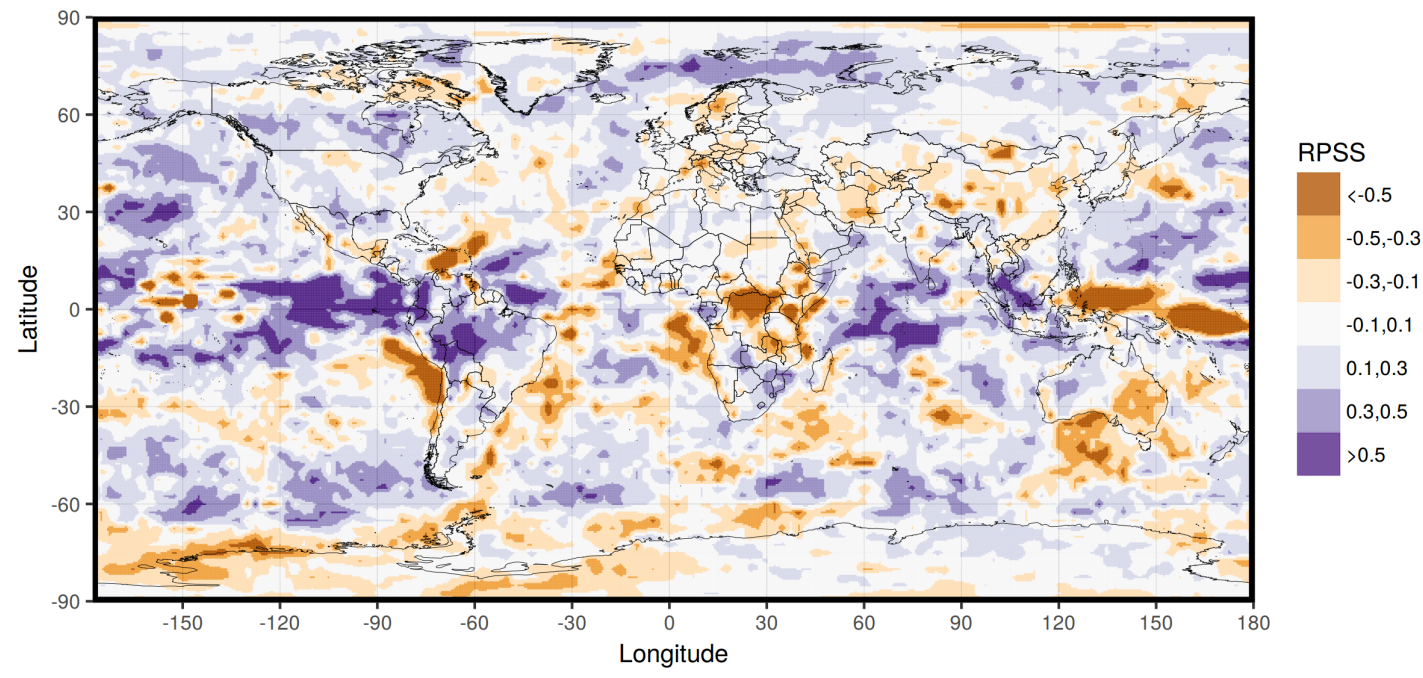

Figure 3: the RPSS score for Glosea5 DJF evaluated using the RPS of Markov Chain as reference forecast instead of the climatology on the period 1996-2009. Map created with R 3.3.0 [15] 

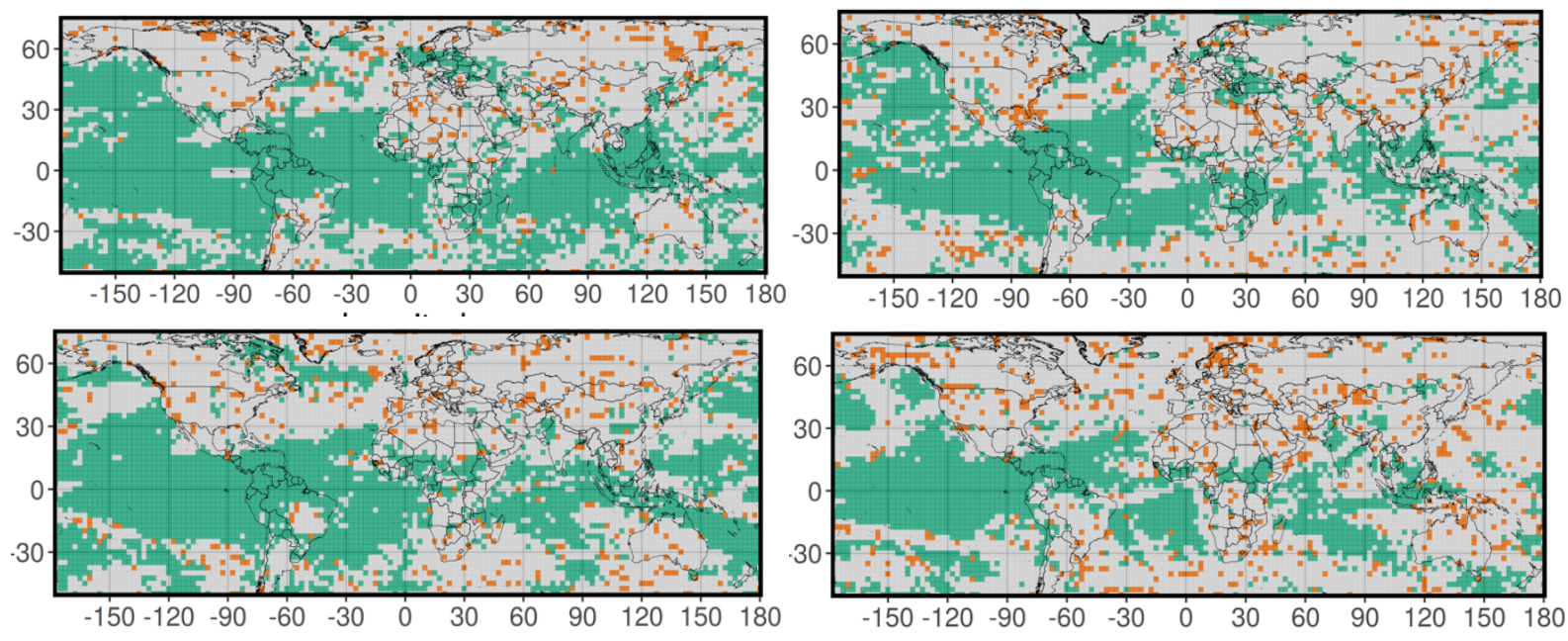

Figure 4: the categorisation of the transition matrices for all the seasons (first row: MAM, JJA; second row: SON, $D J F)$. In orange the 'switching' category, in green the 'persistence' and in grey 'unpredictable'. See section Methods for further details. Map created with R 3.3.0 [15]

\section{Methods}

Observational data sets. Observed temperature data used in this paper has been obtained from the ECMWF's ERA-20C [8] reanalysis, considering the period from 1900 to 2010 . The used data has a resolution of $2.5^{\circ} \times 2.5^{\circ}$ and a seasonal resolution, i.e. four samples each year one for each meteorological season. Low-frequency trends have been removed by using a spline-based approach: for each grid point a cubic smoothing spline [9], with the smoothing parameter chosen by a cross-validation procedure, and then it is removed from the original data to obtain a detrended time-series.

Seasonal forecast data. The seasonal forecast here considered is the UK Met Office's GloSea5 [10] hindcast with 12 members on the period 1996-2010. This data set is available through the ECOMS User Data Gateway (ECOMS UDG, available at http://www.meteo.unican.es/ecoms-udg) and, in spite of the presence of another GloSea5 dataset with more ensemble members (24), it is the only one with four initializations per year instead of two.

For the comparison, the forecast has been interpolated on the same grid of the reanalysis. The skill of the forecasts has been measured using the implementation of Ranked Probability Skill Score (RPSS, see [13]) available in the easyVerification R package [11]. Direct model output has been used without any post-processing procedure, except for interpolation, to correct any bias or drift. The proposed approach, based on the use of tercile categories for seasonal averages at one-month of lead time, is not affected neither by drift nor bias.

Markov-Chain analysis. To test the value of the climate predictions for Europe we design and run a benchmark model based on a simple Markov Chain process. This is a class of memoryless processes designed over a set of arbitrarily defined state. The basic assumption for a process to be Markovian is that the probability of transition from one state to another only depends on the state itself rather than on how it got to that state. Higher order Markov 
processes (which consider step 1,2, ..) are also possible but we have not considered them in current study. We defined the process using the monthly mean value of a meteorological parameter categorised by using seasonal terciles, i.e. the state space of the Markov process consists of three possible states (low, medium, high). To increase the robustness of the presented results we have applied a leave-one-out cross-validation procedure when computing the terciles defining the categories.

Then we looked at the probability of transition from each tercile category to all the other on a seasonal basis. For each season (here: DJF, MAM, JJA and SON) we have measured the conditional probability of going from state $i$ to state $j$, e.g. $P\left(X_{M A M}=j \mid X_{D J F}=i\right)$. For each season, a $3 \times 3$ matrix $P$, called transition matrix, can be formed by those probabilities, considering all the possible transitions (see Fig. 1 for example). This matrix can be used to give a probabilistic forecast selecting the "row" corresponding to the state (i.e. the tercile category) for the season $s$ at the year $t$.

We can classify the transition matrix in three categories: persistence, switching and unpredictable. The classification rules for the transition matrices are the following:

- "persistence": when any of the values on the diagonal are greater than a threshold (here 0.55)

- "switching": when the matrix has not been categorised as "persistence" and when any value not on the diagonal is greater than a threshold (here 0.45)

- "unpredictable": when the matrix has not been classified as "persistence" or "switching"

\section{References}

1. Van den Dool, H. Empirical methods in short-term climate prediction. (Oxford University Press, 2007).

2. van Oldenborgh, G. Searching for decadal variations in ENSO precipitation teleconnections. Geophysical Research Letters 32, (2005).

3. Suckling, E., van Oldenborgh, G., Eden, J. \& Hawkins, E. An empirical model for probabilistic decadal prediction: global attribution and regional hindcasts. Climate Dynamics 48, 3115-3138 (2016).

4. Eden, J., van Oldenborgh, G., Hawkins, E. \& Suckling, E. A global empirical system for probabilistic seasonal climate prediction. Geoscientific Model Development 8, 3947-3973 (2015).

5. Markov, A. Extension of the Limit Theorems of Probability Theory to a Sum of Variables Connected in a Chain. Dynamic Probabilistic Systems (Volume I: Markov Models). Ed. R. Howard. New York City: John Wiley \& Sons, Inc., 1971. chapter Appendix B, 552-577.

6. Gabriel, K. \& Neumann, J. A Markov chain model for daily rainfall occurrence at Tel Aviv. Quarterly Journal of the Royal Meteorological Society 88, 90-95 (1962).

7. Pasmanter, R. \& Timmermann, A. Cyclic Markov chains with an application to an intermediate ENSO model. Nonlinear Processes in Geophysics 10, 197-210 (2003).

8. Poli, P. et al. ERA-20C: An Atmospheric Reanalysis of the Twentieth Century. Journal of Climate 29, 4083-4097 (2016).

9. Chambers J., Hastie T., Pregibon D. Statistical Models in S. In: Momirović K., Mildner V. (eds) Compstat. Physica-Verlag HD (1990)

10. MacLachlan, C. et al. Global Seasonal forecast system version 5 (GloSea5): a high-resolution seasonal forecast system. Quarterly Journal of the Royal Meteorological Society 141, 1072-1084 (2014).

11. MeteoSwiss, easyVerification: Ensemble Forecast Verification for Large Data Sets. R package version 0.3.0. (2016)

12. Scaife, A. et al. Skillful long-range prediction of European and North American winters. Geophysical Research Letters 41, 2514-2519 (2014).

13. Wilks, D. Statistical methods in the atmospheric sciences. (Academic Press, 2006). 
14. Smith, L., Du, H., Suckling, E. \& Niehörster, F. Probabilistic skill in ensemble seasonal forecasts. Quarterly Journal of the Royal Meteorological Society 141, 1085-1100 (2014).

15. R Core Team (2017). R: A language and environment for statistical computing. R Foundation for Statistical Computing, Vienna, Austria. (https://www.R-project.org/)

\section{Contributions}

C.B. developed the idea that led to this paper. M.D.F. implemented the models, designed the data visualization. C.B. and M.D.F. analysed the data and wrote the main paper.

\section{Competing financial interests}

The authors declare no competing financial interests.

\section{Corresponding authors}

Correspondence to Matteo De Felice or Carlo Buontempo

\section{Acknowledgements}

The research leading to these results has received funding from the EUPORIAS project, funded by the European Union's $7^{\text {th }}$ Framework Programme for research, grant agreement 308291 . We are grateful to Anca Brookshaw for the very useful comments she provided to an early version of the manuscript 